\title{
Performance Analysis of Dispersion Compensation in Long Haul Optical Fiber with DCF
}

\author{
Saurabh Kumar ${ }^{1}$, Prof. A. K. Jaiswal ${ }^{2}$, Er. Mukesh $\mathrm{Kumar}^{3}$, Er. Rohini Saxena ${ }^{4}$ \\ ${ }^{1}$ (M.Tech Student of ECE, SSET/ SHIATS, Allahabad, India) \\ ${ }^{2}$ (Prof. \& Head of ECE, SSET/ SHIATS, Allahabad, India) \\ ${ }^{3,4}$ (Assistant Prof. of ECE, SSET/ SHIATS, Allahabad, India)
}

\begin{abstract}
In this paper, We investigate post, pre and symmetrical/mix dispersion compensation methods for 40 Gb/s non-return to zero link using standard and dispersion compensated fiber through $F B G$ compensator to optimize high data rate optical transmission. The influence of dispersion of FBG compensator and increases in the power of $C W$ laser has been studied to evaluate the performance of optical communication systems. The simulation model of the WDM based on the Optisystem 11.0 is presented according to the above principle. The simulation results are validated by analysing the $Q$-factor and Bit error rate (BER) in the numerical simulator. It is found that post compensation performance is best and the input fiber power is taken as 5-10dB, the corresponding BER performance is better.
\end{abstract}

Keywords - BER, Dispersion compensation, Q-factor, WDM.

\section{Introduction}

When optical signals are transmitted over optical links, different wavelength components of the optical signals will generally experience different propagation times due to the fact that the transport medium has different effective refractive indices for different wavelengths. In recent years, with the rapid growth internet business needs, people urgently need more capacity and network systems. So the demand for transmission capacity and bandwidth are becoming more and more challenging to the carriers and service suppliers. Under the situation, with its huge bandwidth and excellent transmission performance, optical fiber is becoming the most favourable delivering media and laying more and more important role in information industry $[2,3]$.

Therefore, it is very necessary to investigate the transmission characteristics of optical fiber. And the main goal of communication system is to increase the transmission distance. Loss and dispersion are the major factor that affect fiber-optical communication being the high-capacity develops. The EDFA is the gigantic change happened in the fiber-optical communication system; the loss is no longer the major factor to restrict the fiber optical transmission. Since EDFA works in $1550 \mathrm{~nm}$ wave band, the average Single Mode Fiber (SMF) dispersion value in that wave band is very big, about $15-20 \mathrm{ps} /(\mathrm{nm} . \mathrm{km}-1)$. It is easy to see that the dispersion become the major factor that restricts long distance fiber optical transfers.[4]. In this study, we propose three DCF compensation scheme, post-compensation, pre-compensation and symmetrical/mix compensation scheme. Simulation studies show that post compensation scheme is the best. It can greatly reduce the influences of the fiber nonlinearity and increase the transmission distance greatly.

The rest of paper is organized as followed. In Section II, the cause of fiber dispersion and its effects on optical transmission is introduced. In Section III, present fiber dispersion compensation technology. In Section IV, present the research of DCF dispersion compensation scheme. Section V present transmission system simulation with OPTISYSTEM 11.0, analyses and compare each kind of compensation scheme, Section VI concludes the paper.

\section{Fiber Optic Dispersion On Optical Transmission}

Loss and dispersion are the major factor that affect fiber-optical communication being the highcapacity develops. The EDFA is the gigantic change happened in the fiber-optical communication system; the loss is no longer the major factor to restrict the fiber-optical transmission. Since EDFA works in $1550 \mathrm{~nm}$ wave band, the average Single Mode Fiber (SMF) dispersion value in that wave band is very big, about 15-20ps / (nm. $\mathrm{km}^{-1}$ ). It is easy to see that the dispersion become the major factors that restrict long distance fiber-optical transfers. Dispersion is defined as because of the different frequency or mode of light pulse in fiber transmits at different rates, so that these frequency components or models receive the fiber terminals at different time. It can cause intolerable amounts of distortions that ultimately lead to errors. In single-mode fiber performance is primarily limited by chromatic dispersion which occurs because the index of the glass varies slightly depending on the wavelength of the light, and light from real optical transmitters necessarily has nonzero spectral width. Polarization mode dispersion another source of limitation occurs because although the single-mode fiber can sustain only one transverse mode, it can carry this mode with two different polarizations, and slight 
imperfections or distortions in a fiber can alter the propagation velocities for the two polarizations. This phenomenon is called birefringence. Mode birefringence $B_{m}$ is defined as the follow

$$
\mathrm{B}_{\mathrm{m}}=\frac{\beta x-\beta y}{k 0}=\mathrm{n}_{\mathrm{x}}-\mathrm{n}_{\mathrm{y}}
$$

Where nx, ny are the effective refractive of the two orthogonal polarizations. For a given $\mathrm{Bm}$, its fast axis and slow axis components will be formed the phase difference after the light waves transmission L Km.

$$
\varphi=\mathrm{k}_{0} \mathrm{~B}_{\mathrm{mL}} \frac{2 \pi}{\lambda}\left(\mathrm{N}_{\mathrm{X}}-\mathrm{N}_{\mathrm{Y}}\right)=(\beta x-\beta y) \mathrm{L}
$$

If the $\mathrm{B}_{\mathrm{m}}$ is a constant, through the light waves in transmission process the phase difference between its fast axis and slow axis will periodicity repetition. The length that it leads to a phase difference of $2 \pi$ or power periodic exchange is called polarization beat length.

$$
\mathrm{L}_{\mathrm{B}}=\frac{2 \pi}{\beta x-\beta y}=\frac{\lambda}{\beta m}
$$

If the incident light has two polarization components, due to refractive difference between the fast axis and slow axis, the transmit rate of two polarization components will be different. Degree of pulse broadening can be expressed by different group delay $\Delta \tau$. The influence of dispersion on system performance is also reflected in the optical fiber nonlinear effects. Dispersion increased the pulse shape distortion caused by the selfphase modulation dispersion (SPM); the other hand, dispersion in WDM systems can also increase the crossphase modulation (XPM), four-wave mixing (FWM) and other nonlinear effects [6,7].

\section{Dispersion Compensation Technology}

To improve overall system performance and reduced as much as possible the transmission performance influenced by the dispersion, several dispersion compensation technologies were proposed. Amongst the various techniques proposed in the literature, the ones that appear to hold immediate promise for dispersion compensation and management could be broadly classified as: dispersion compensating fiber (DCF), chirped fiber Bragg gratings (FBG), and high-order mode (HOM) fiber [8]. The idea of using dispersion compensation fiber for dispersion compensation was proposed as early as in 1980 but, until after the invention of optical amplifiers, DCF began to be widespread attention and study. DCF has become a most useful method of dispersion compensation and has been extensively studied.

There is positive second-order and third-order dispersion value in SMF while the DCF dispersion value is negative. So by inserting a DCF, the average dispersion is close to zero. As the local dispersion of higher transmission link, FWM and XPM were ignored; only to consider the role of SPM and dispersion.

\section{Dispersion Compensation Scheme}

To support a high-capacity wavelength-division-multiplexing (WDM) transmission, the embedded standard single-mode fiber (SMF) should be up graded to overcome the dispersion limit. In this paper dispersion compensating fiber is analyzed with dispersion compensation with the help of fiber bragg compensator. According to relative position of DCF and single mode fiber, post-compensation, pre-compensation and symmetrical/mix compensation is proposed. DCF Pre-compensation scheme achieve dispersion compensation by place the DCF before a certain conventional single-mode fiber, or after the optical transmitter. Post compensation scheme achieve dispersion compensation by place the DCF after a certain conventional singlemode fiber, or before the optical transmitter. Symmetrical/mix compensation scheme is consist of post compensation and pre compensation. Different location on the system will generate different nonlinear effects. The Simulation of three dispersion compensation system is shown in Figure 1.

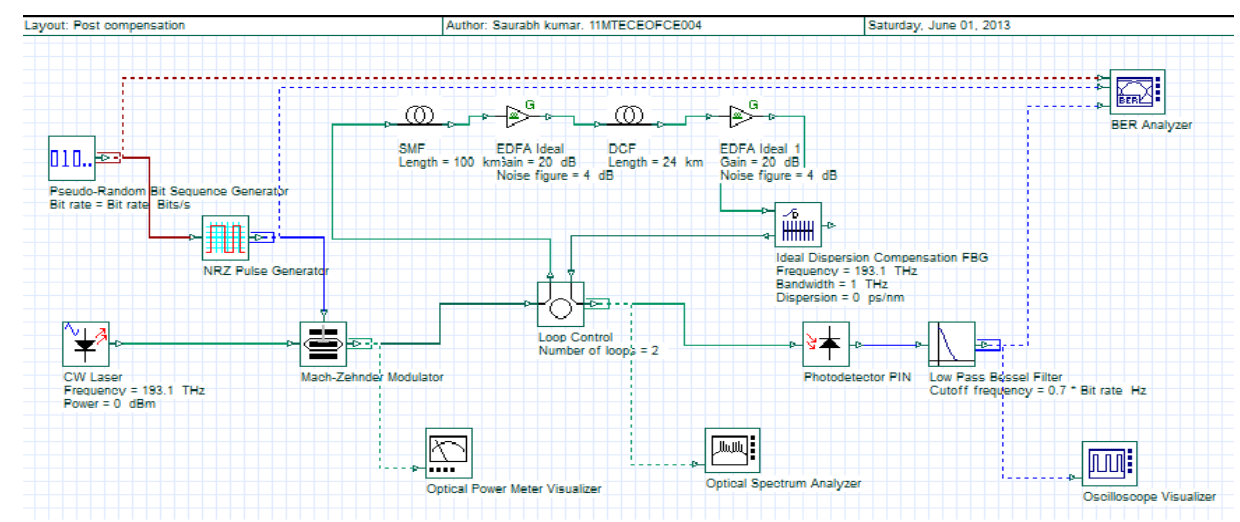

(a) Simulation for post compensation 


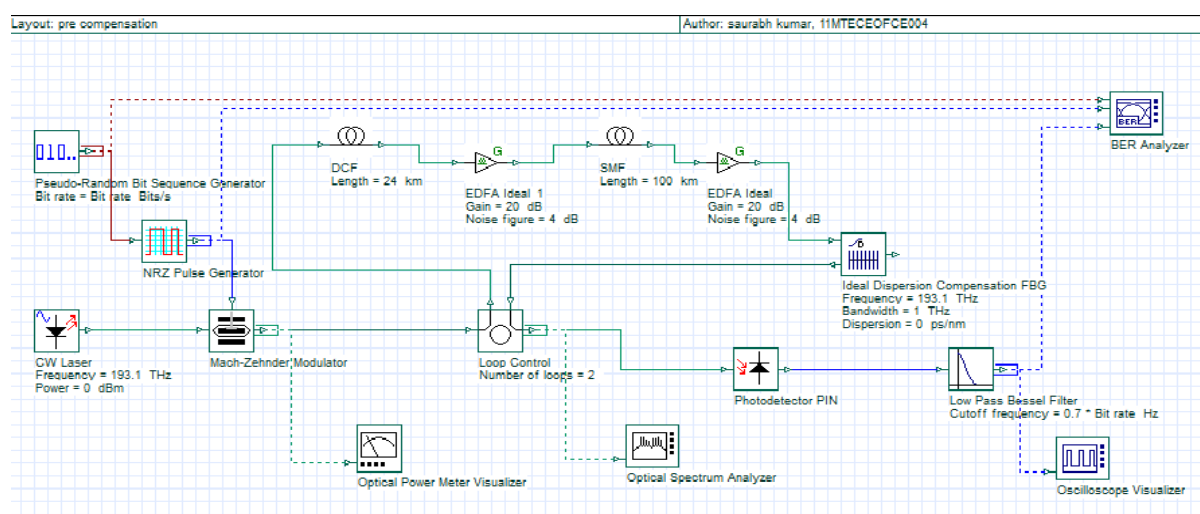

(b) Simulation for pre compensation

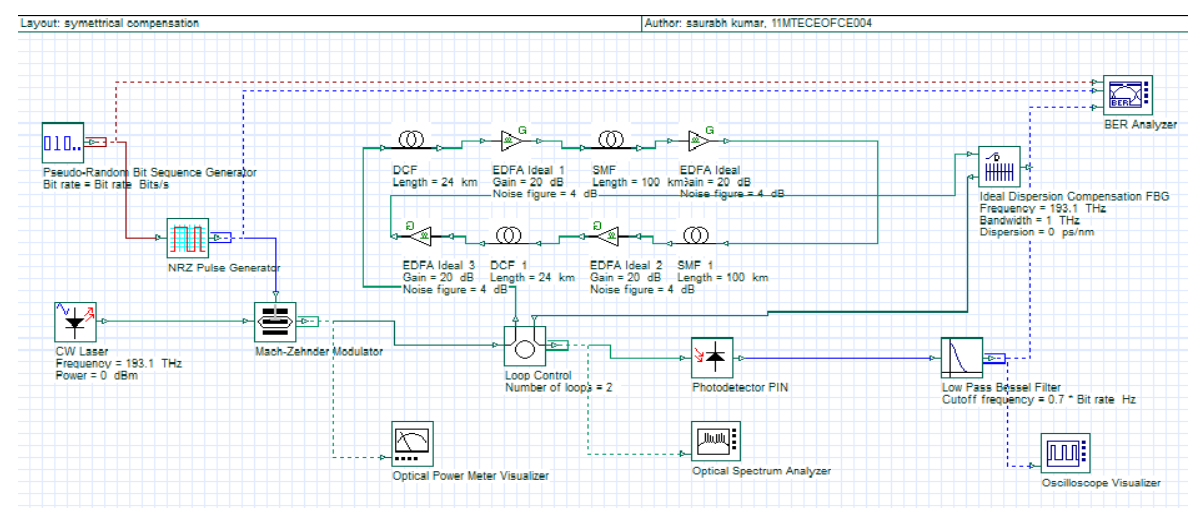

(c) Simulation for symmetrical compensation

Figure 1 : Three dispersion compensation scheme

Figure 1 (a) shows the post compensation schemes. The simulation module includes the transmission module, transmission link and the receiver module. Simulation model use Mach-Zehnder external modulator to modulate the CW Laser at central frequency 193.1 THz. Optical fiber transmission link composed of a 100Km. The kind of optical fiber is G.655. Dispersion compensation is achieved with fiber bragg gratings (FBG) are implemented instead of using dispersion compensated fiber (DCF). EDFA is used to compensate the power loss generating by SMF and the DCF signal. Receiver module includes de multiplexer and receiver filters.

Figure 1 (b) and Figure 1 (c) respectively show the pre compensation scheme and the symmetrical/mix compensation scheme. The simulation configuration is as similar as Figure 1 (a). The difference is that at the transmitter DCF compensate $100 \mathrm{Km}$ of single-mode fiber dispersion in Figure 1 (b). In Figure 1 (c), there is a symmetrical/mix compensation scheme.

\section{Result And Analysis}

In optical communication only optical signal to noise ratio (OSNR) could not accurately measure the system performance, especially in WDM systems. Quality factor is a one of the important indicators to measure the optical performance by which to characterize the BER. Figure 2 and Figure 3 display the influence of input optical power on the performance of transmission system.

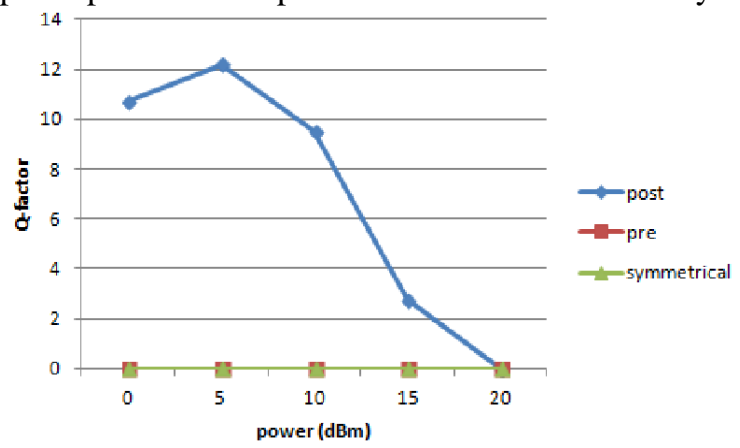

Figure 2: Comparison among three dispersion compensation (DCF) on the basis of Q-factor

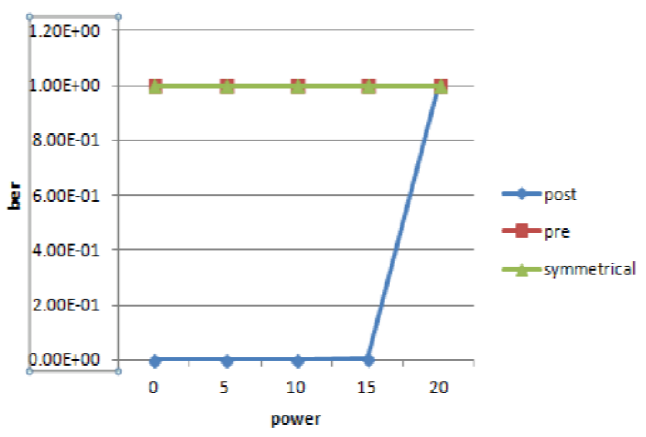

Figure 3: Comparison among three dispersion compensation (DCF) on the basis of BER 
Figure 2 shows the effect on Q-factor with the variation in optical power and compensator dispersion in fiber bragg grating (FBG) of all dispersion compensation fiber (DCF) model. We can observe that with the input power increasing steadily up to about $5 \mathrm{dBm}$ brings the nominal increases in Q-factor and when input optical power approaches to $5 \mathrm{dBm}$, the Q-factor becomes maximum. When equal power is launched into the various models of the link, the Q-factor of post compensation scheme is greater than the two other kinds of dispersion compensation scheme. When the input optical power is greater than $5 \mathrm{dBm}$ the quality factor decreased gradually and the error performance is gradually degraded. This is because as the optical power increases, nonlinear effects increase, but the optical signal noise ratio increased. Through the whole system study found that the performance of post compensation system is best in the long-distance high-speed WDM systems.

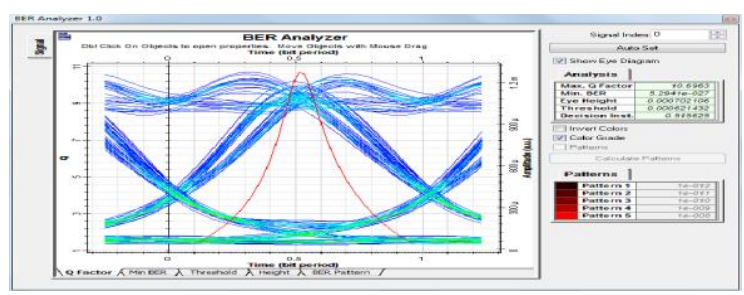

(a) At $0 \mathrm{~dB}$ power with compensator dispersion $-2000 \mathrm{ps} / \mathrm{nm}$ for post compensation

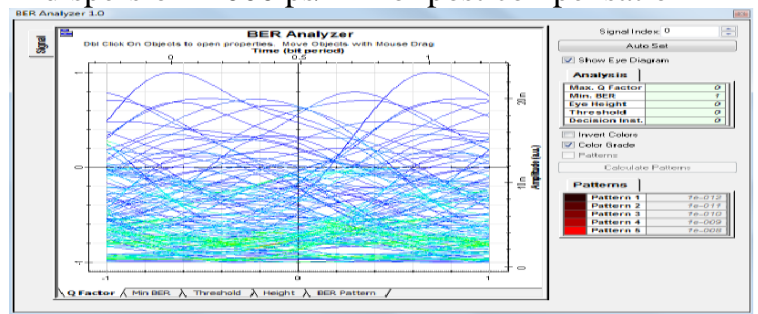

(b) At $0 \mathrm{~dB}$ power with compensator dispersion $-2000 \mathrm{ps} / \mathrm{nm}$ for pre compensation

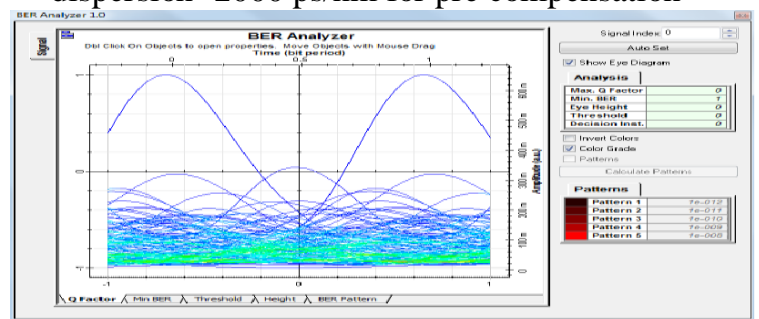

(c) At $0 \mathrm{~dB}$ power with compensator dispersion $-2000 \mathrm{ps} / \mathrm{nm}$ for symmetrical compensation

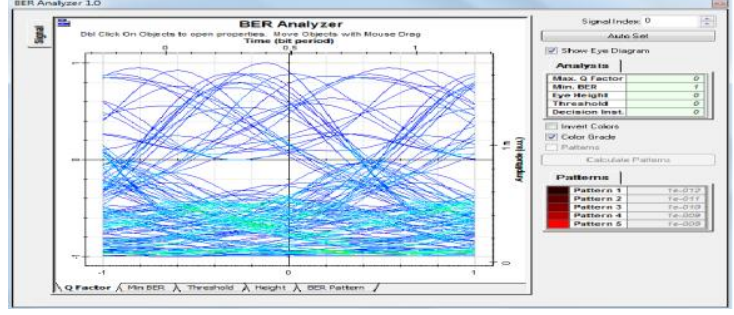

(d) At $0 \mathrm{~dB}$ power with compensator dispersion $0 \mathrm{ps} / \mathrm{nm}$ for post compensation

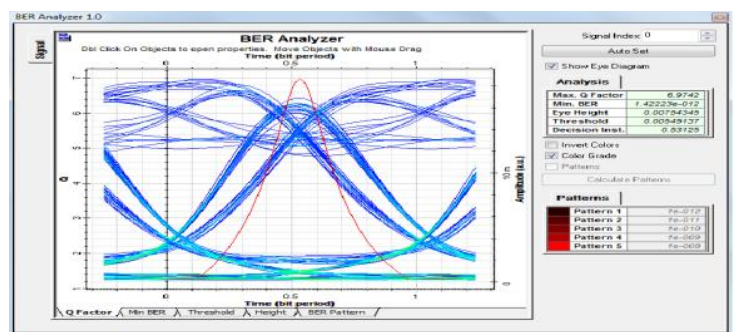

(e) At $0 \mathrm{~dB}$ power with compensator dispersion $0 \mathrm{ps} / \mathrm{nm}$ for pre compensation

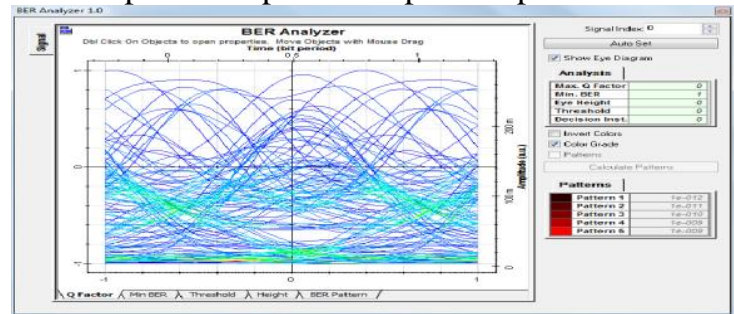

(f) At $0 \mathrm{~dB}$ power with compensator dispersion $0 \mathrm{ps} / \mathrm{nm}$ for symmetrical compensation

Figure 4: Eye diagram of different compensation technique at different compensator dispersion of fiber bragg

Figure 4 gives the simulation results at different dispersion compensator of fiber bragg at same optical power. As we can see from the figure 4 (a), the effect of dispersion compensation is very good with $2000 \mathrm{ps} / \mathrm{nm}$ dispersion compensator of fiber bragg at $0 \mathrm{~dB}$ power. The signal quality is high, eye's shape is very good and the effect of dispersion compensation is quite good. The curve of Q-factor changes with the open degree of eye diagram as follows. More approaches the point of largest eye diagram opened, the Q-factor is bigger, and the corresponding BER is smaller.

\section{Conclusion}

On the basis of compared and analyzed the three system simulation results conclusions are as the followings. Using fiber bragg gratings (FBG) are implemented instead of using dispersion compensated fiber (DCF) for dispersion compensation in $40 \mathrm{~Gb} / \mathrm{s}$ WDM system is an effective solution. It is observed that the compensation schemes reduced the dispersion appropriately but among them post compensation scheme reduced the accumulated fiber chromatic dispersion to the maximum possible extent. A moderate lesser value of fiber bragg grating dispersion and bigger value of laser average power is favorable to the performance of the transmission system. Variation of the input power launched and compensator dispersion of fiber bragg grating resulted in variation in Q-factor and the corresponding graph showed that at $5 \mathrm{dBm}$ and $-2000 \mathrm{ps} / \mathrm{nm}$ the link performance with the regard the Q-factor provides the better result. 


\section{References}

[1] Bo-ning HU, Wang Jing, Wang Wei, Rui-mei Zhao, “Analysis on Dispersion Compensation with DCF based on Optisystem”. 2nd International Conference on Industrial and Information Systems pp. 40-43 2010

[2] Mochida Y, Yamaguchi N, Ishikawa G, "Technology-oriented review and vision of 40Gb/s based optical transport Networks", Journal of light-wave technology.PP. 2272-228,12002,20(12)

[3] Omae T, "Universal conditions for estimating the nonlinear refractive index $\mathrm{n}_{2}$ of dispersion com- pensating fibers by the CWSPM method", IEEE Photon. Technol. Lett., Vol 13. No.6, pp. 571-573, Nov, 2001.

[4] Zou X Y, Hayee M I, H wang S M, et al. Limitations in 10Gb/s WDM optical-fiber transmission when using a variety of fiber types to manage dispersion and nonlinearities[J]. Lightwave Technol., PP: 1144-1152,June,1996.

[5] BU CHAL IF, LANNES. Fast eye monitor for 10G/bs and its applicationg for optical PMD compensation [Z]. Optical Society of America,2000.

[6] Killy R I, Thiele H J, Mikhailov v, ea al. Reduction of intrachannel nonlinear distortion in 40-Gb/s based WDM transmission over standard fiber [J]. IEEE Photonics Technology Letters, 2000, 12(12): 1642-1626.

[7] Eggleton B 1. Dynamic dispersion, compensation devices for high speed transmission systems. Optical Fiber communication conference and exhibit, 2001(3): WHIII-WH1I3

[8] jianjun Yu, Bojun Yang,"Dispersion-allacated soliton technology with long amplifier spacing and long distance," IEEEphoton technol lett, vol 9, pp. 952-954,No.7, 1997. 